\title{
DESIGN AND IMPLEMENTATION OF A LOW-COST NETWORKING AND VOIP LAB FOR UNDERGRADUATE NETWORKING CURRICULUM INSTRUCTION
}

\author{
Tom Imboden, Southern Illinois University-Carbondale, timboden@ siu.edu \\ Derek Strothmann, Southern Illinois University-Carbondale, dtstroth@ siu.edu
}

\begin{abstract}
Traditional networking curriculum emphasizes the use of tools like Ping and Traceroute in order to test and verify connectivity during networking laboratory exercises. The authors argue that the use of inexpensive hardware and freely available open source Voice over Internet Protocol (VoIP) software can provide students in undergraduate networking courses the opportunity to learn about a type of networking application (VoIP) oftentimes overlooked during coursework while providing them with an insight into a practical application to install on and test on networks. This paper provides a framework for the creation of a low-cost lab for networking curriculum instruction to be utilized in a classroom setting.
\end{abstract}

Keywords: VoIP, Network Administration, Architecture Framework

\section{INTRODUCTION}

There are countless textbooks, web sites, and electronic study guides aimed at teaching students the fundamentals of local area networks (LANs), wide area networks (WANs), and the Transmission Control Protocol/Internet Protocol (TCP/IP) suite. One common thread tying these sources together is the use of tools such as Ping and Traceroute as the exclusive means of verifying connectivity during the course of hands-on laboratory exercises. While these tools are certainly invaluable, solely using them for connectivity testing and verification does not present the student with the full application of the TCP/IP or OSI network models and may seem dry or fail to present the students with application level consequences and effects to network changes. The contribution of this paper is the presentation of a means of creating a flexible, capable, and inexpensive networking laboratory to teach and demonstrate LAN, WAN, and VoIP fundamental concepts.

In this paper, we first present a brief review of literature discussing similar projects where networking labs, courseware, and curriculum have been designed, implemented, and evaluated. Second, we provide details regarding the laboratory design and discuss the relevant technologies and equipment used for the creation of the low-cost lab as well as provide approximate pricing and alternative equipment. Third, we present an argument for using inexpensive VoIP technology over networking equipment used in hands-on lab exercises as a means of demonstrating successful network configuration and connectivity and provide qualitative student data supporting the use. We believe students benefit from the exposure and experience working with VoIP technology in a lab course setting. An example of how fundamental networking concepts outlined in the 2008 SIGITE IT curriculum can be demonstrated and exhibited in conjunction with the lab equipment will motivate the use of the low-cost lab in networking courses. Suggestions for improving the lab environment, incorporating additional application layer network components, and measuring student learning as part of future research follows. Finally, we conclude the paper.

\section{LITERATURE REVIEW}

A review of literature leads the authors to believe that this project is the first of its kind, blending LAN, WAN, and VoIP technologies into a single laboratory framework for an undergraduate networking curriculum. Other authors have discussed the creation of a hands-on networking laboratory [7], creating courseware based on open source material [6], and generalized networking lab configurations geared towards the teaching of LAN technologies $[1,3,5]$. Absent from the literature is the discussion of instruction of WAN implementation, administration, and troubleshooting using VoIP as an application layer test device.

As far back as 1995, academics have been considering the benefits of creating hands-on laboratory exercises for students in computer networking courses. Mengel and Bowling [5] identify the benefit of exposing students to networking equipment in a structured setting as part of both undergraduate and graduate networking courses. Their paper also documents that faculty 
believed that exposing students to a variety of operating systems including Windows, Linux, and NetWare, during their networking coursework was beneficial. More recent literature has reinforced this same belief. Cigas discusses a similar approach, utilizing multiple computers with network switches and routers to create small networks which connect to the laboratory's backbone network in order to study three general categories: networking, system administration, and security [3].

The authors of Hansen et al. [4] also write about implementing laboratory exercises during a concentrated, nine-day course teaching students about open source VoIP technologies, but omit IPbased WAN technologies as part of their discussion. Their course objectives combine both lecture and laboratory work while teaching students about protocols, tools, and applications used on the open source IP telephony platform, Asterisk.

The Special Interest Group for Information Technology Education's (SIGITE) 2008 curriculum guidelines for undergraduate Information Technology degree programs [2] lists networking as one of thirteen knowledge areas in their recommended fouryear IT curriculum. The networking area is broken into six units, which can be thought of as subareas of networking instruction. They are listed on page 98 of the guidelines document and further details regarding recommended instruction for each are provided.

\section{Foundations of Networking \\ 2. Routing and Switching \\ 3. Physical Layer \\ 4. Security \\ 5. Network Management \\ 6. Application Areas}

An institution seeking to align its curriculum with the SIGITE 2008 guidelines will ensure the requisite skills and technologies are taught in appropriate IT courses. In addition to providing content recommendations for IT programs, the 2008 SIGITE guideline also states that "Information Technology is a laboratory discipline"(page 59) [2] and that costs for IT education are "by no means limited to hardware", even though networking devices and equipment "have become commodity items, which makes the hardware far more affordable" [2]. As funding for lab equipment becomes more difficult to obtain for educational institutions, it is clear that stretching every precious dollar as far as possible is desirable. Reusing equipment, leveraging discount hardware avenues, and employing open source and freely available software doesn't necessarily have to impact the student's education negatively.

\section{LAB CONSTRUCTION}

The construction of the lab is relatively straight forward and is designed to mimic how several distributed "corporate offices" connect to each other and can communicate using a WAN. In the classroom our lab is constructed across four rows with three or four students in each row. The equipment dedicated to each row consists of workstations that have been "handed-down" from other school labs, networking devices donated from local businesses or given as surplus from the university IT department, purchased cheaply from auction web sites, or bought new. The networking devices are mounted in two-post, half-height telecom racks with one per row. A picture of one of the racks can be seen in Figure 1.

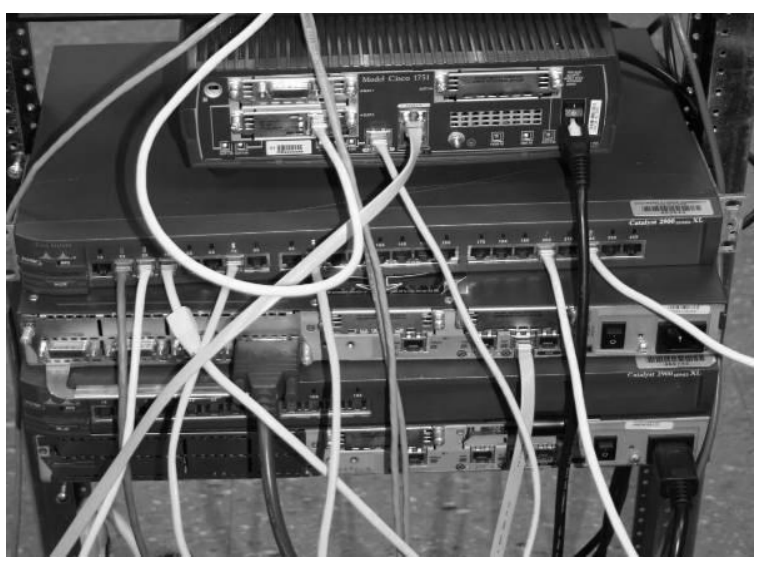

Figure 1 - Example of Row Equipment

It should be noted that the lab environment networking hardware outlined in this paper consists almost exclusively of Cisco branded equipment. While some may argue this limits the students' exposure to the full range of existing network equipment, it does present certain advantages. First, older and typically inexpensive routers, switches, and firewalls are widely available on Internet auction sites. Secondly, an expansive range of reference materials for Cisco hardware and software is also abundant and easy for students to purchase. Finally, by standardizing the equipment manufacturer across the lab, it allows students to focus on learning (and instructors on teaching) the fundamental concepts of networking rather than the quirks and differences of dissimilar manufacturer's equipment and operating systems.

At the heart of each team's equipment rack is a single, 24 port layer two switch. Our environment 
contains Cisco Catalyst 2924 switches. These allow for implementation of VLANs and assigning ports to them in order to create multiple separate layer two network segments. This permits further subnetting and breaking each row up into separate subnets when learning about variable length subnet masks (VLSM) and other types of network segmentation. These types of switches are end-of-life, however, are still useful and relatively inexpensive. They can regularly be purchased on auction sites for as little as \$25. As only very rudimentary switching functionality is needed for basic LAN and WAN coursework, these particular models or others that allow for creation of layer two VLANs and VLAN trunking are suitable. If funding exists, comparable switches from major manufacturers can be purchased new for around $\$ 175$. It is important to use switches with enough ports to support multiple workstations, connections to routers, and VoIP phones to connect. Ideally, each row should utilize the same model of switches, so instructional material for switch configuration can be consistent.

Secondly, a router is necessary to connect the row environment LAN to the WAN infrastructure or the "cloud." As there are two separate networks in this specific scenario, the chosen router must have two Ethernet ports. Our lab employs Cisco 1750 modular routers which contain one fixed Fast Ethernet port, a secondary interface card with a single Ethernet port, and a serial interface card. The routers are commonly available on auction sites for under $\$ 30$, the Ethernet cards for $\$ 35$, and serial interface cards for $\$ 25$. There are certainly alternatives to using Cisco routers. However, these particular models are readily available at low-cost and can make use of a wide variety of interface cards to demonstrate network types and layer two protocols other than Ethernet. It is important to note that a range of serial interface cards exist, many with unique connectors, and that care should be taken when purchasing serial interface cards and cables to ensure the required types are obtained. Another option is to utilize a multi-homed, older PC to function as a router. Ethernet cards are inexpensive, and can be added to a PC to create a router using one of many freely available Linux distributions. Even "Live CDs" specifically designed for creating low-cost routers exist.

In the lab environment, two additional Cisco routers are employed in order to create more advanced networking scenarios. These consist of various 2500 series and 2600 series routers. These routers are also available at minimal cost, as low as $\$ 10$ - \$20, and contain Ethernet, serial, and other network interfaces for students to experiment with configuring. A plethora of modular interface cards for T1 circuits, serial, Integrated Service Digital Network (ISDN), and other network connection types are available inexpensively and can be added to provide supplemental connectivity options.

Each lab row contains three or four workstations for student use. The PCs used are older model Dell Optiplex desktops with $40 \mathrm{~GB}$ hard drives, $512 \mathrm{MB}$ RAM, and Celeron CPUs. The operating system installed is Windows XP, as they were shipped with and licensed for it. A secondary network card has been added to allow for maximum network flexibility. A range of free software applications are installed to perform various networking functions, tasks, administration, and testing. Putty is used for remote telnet, Secure SHell (SSH), and console connectivity (www.putty.org). SolarWinds Trivial File Transfer Protocol (TFTP) Server is installed and available to function as a TFTP server when backing up or installing device configurations or operating systems (www.solarwinds.com). Sun's Open Office is a free suite for creating word processing documents, spreadsheets, presentations, and basic drawings (www.openoffice.org). If Windows is not available, any number of free Linux distributions can be substituted and will provide the same functionality for student workstations. These PCs are also used as softphones, or VoIP clients that provide voice connectivity to a VoIP server with the use of a headset/microphone combination. There are numerous free softphones available for Windows and Linux. The one chosen for our environment is Counterpath's X-Lite (www.counterpath.com). This particular application was picked due to its ability to support video traffic in addition to voice. For Linux machines, a suitable VoIP client is Twinkle (www.xs4all.nl/ mfnboer/twinkle).

A separate PC workstation is used for the installation of the VoIP server software, trixbox (www.trixbox.org) (officially spelled with lowercase ' $t$ '). The hardware requirements for the trixbox are minimal, and it can easily be installed on an additional machine of the type described in the previous paragraph. Another alternative, if more powerful machines are available, is to install trixbox as a virtual machine using any of the freely available virtual environments. These include VMware Server (www.vmware.com) Sun VirtualBox (www.virtualbox.org), and Microsoft Virtual PC (www.microsoft.com/windows/virtual-pc/). The trixbox server provides registration, admission, and status (RAS) for clients. Phones register using the Session Initiation Protocol (SIP) so the trixbox server 
is aware the client is ready to place and receive calls and the IP address from which it registered. The trixbox server also provides a wealth of other advanced calling features including automated attendants, intercom, call detail records, conference bridging, roaming extensions, voicemail, trunking to other servers, gateway support, and many other attributes seen in other VoIP-based and traditional enterprise telephony systems.

In addition to softphone VoIP clients, the lab contains several hardware IP phones. These look like standard business-class telephones but connect to IP networks via Ethernet cables. The particular model chosen for our lab is the Grandstream GXP-2000 (www.grandstream.com). These can be purchased new for around $\$ 75$ each, support up to four separate SIP accounts, and contain an auxiliary switch port to allow another Ethernet device to connect to the LAN segment. They also provide support for networking and security features such as 802.3af Power-overEthernet (PoE) and MD5 hashing. It is the authors' belief that providing students with the physical hardware IP phones is beneficial, as it affords them an opportunity to work with embedded firmware as well as web-based portals to configure the required VoIP account and IP settings.

The final component in the lab environment is a multiport layer three router or switch to connect the individual rows and teams in a simulated WAN cloud. This device can be the most expensive, as adding IP routing capability to network hardware typically increases the cost dramatically. However, there are economical alternatives. The hardware employed in our lab is an Extreme Networks Summit 48si, layer three switch (www.extremenetworks.com). These particular models provide layer three IP routing capabilities and routing protocols such as Routing Information Protocol (RIP), Open Shortest Path First (OSPF), and

\section{Figure 2 - Lab Topology}

Border Gateway Protocol (BGP). The Summit 48si also supports the use of Access Control Lists (ACLs), VLANs, Secure SHell (SSH), and Simple Network Management Protocol (SNMP). The switch can be found on auction sites for around $\$ 100$. It is important to note that software licensing of the switch firmware may allow or disallow specific features. Alternative devices to provide connectivity to individual teams exist in the form of routers, other layer three switches, and custom built Linux PC routers. It is imperative that they contain enough Ethernet interfaces to support the number of separate lab teams in an environment. It is important to note that the configuration procedure and commands for the Summit 48 si are significantly different than Cisco IOS-based commands. Because of this, the instructor in our environment is responsible for the configuration and maintenance of the WAN cloud provided by the layer three switch.

The initial VoIP environment is configured in the lab to allow for internal telephony communication within a row. The trixbox servers are assigned static IP addresses on the subnet assigned to the row. Students are responsible for assigning the workstations and hardware IP phones appropriate IP addresses, subnet masks, and gateways and must be aware how these might be affected as hardware is changed or subnet IP schemes are modified. When further subnetting the row networks, these can be altered as required to allow for distributing PCs and IP phones across separate subnets. Logical SIP trunks are created between trixbox servers across the WAN cloud. This allows for voice communication between each of the rows for which a trunk is configured, and the ability to verify network connectivity at all layers.

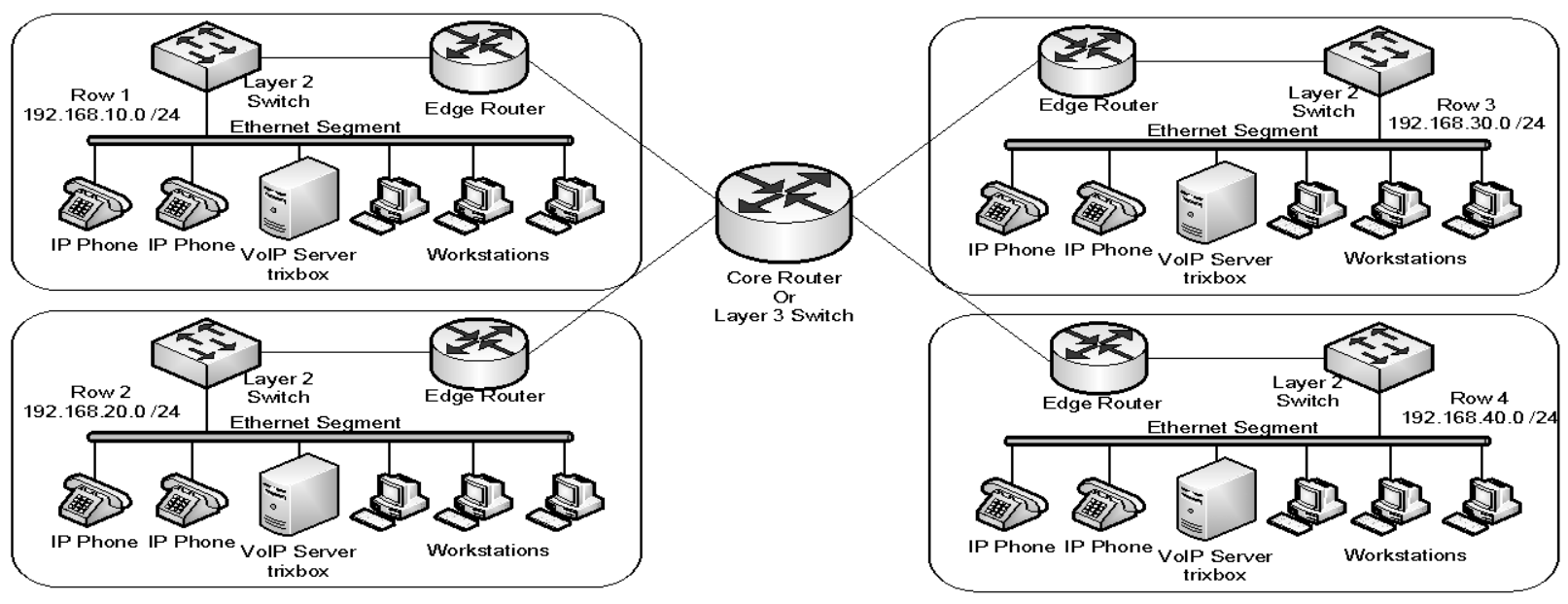

Volume XI, No. 2, 2010

Issues in Information Systems 
The physical topology of the complete classroom lab environment is seen in Figure 2. A second image, Figure 3, illustrates one possible configuration of an individual team's networking equipment using VLSM and serial interfaces to divide and connect a Class C network into multiple smaller networks and use static or dynamic routing protocols to support routing at the network layer. It should be noted that the solid lines in Figure 2 all connect to a single switch but represent separate VLANs on that switch as indicated in the drawing.

As funding for lab equipment is a real concern at many institutions, the main objective and contribution of this paper is to outline and guide those interested in creating a hands-on networking laboratory at minimal cost without sacrificing realistic and useful functionality. As equipment costs can vary on auction, retail, and surplus sites, specific prices for items used may fluctuate. A breakdown of the cost to equip a single row or team in our lab is listed in Table 1 and totals \$300. Across our entire classroom lab environment containing four teams, the total lab hardware cost was approximately $\$ 1300$ as seen in Table 2.

\begin{tabular}{|l|r|r|r|}
\hline \multicolumn{1}{|c|}{ Item } & \multicolumn{1}{c|}{ Qty } & \multicolumn{1}{c|}{ Cost } & \multicolumn{1}{c|}{ Total } \\
\hline Workstations (surplus) & 4 & $\$ 0$ & $\$ 0$ \\
\hline IP Phones & 2 & $\$ 75$ & $\$ 150$ \\
\hline Cisco $1700 \mathrm{w} /$ cards & 1 & $\$ 90$ & $\$ 90$ \\
\hline Cisco 2500 & 1 & $\$ 20$ & $\$ 20$ \\
\hline Cisco 2600 & 1 & $\$ 20$ & $\$ 20$ \\
\hline Cables - misc & - & $\$ 20$ & $\$ 20$ \\
\hline Total for one row/team & & & $\$ 300$ \\
\hline
\end{tabular}

Table 1 - Equipment Cost per Row/Team

\begin{tabular}{|l|r|r|r|}
\hline \multicolumn{1}{|c|}{ Item } & \multicolumn{1}{|c|}{ Qty } & Cost & Total \\
\hline $\begin{array}{l}\text { Equipment for one } \\
\text { row/team }\end{array}$ & 4 & $\$ 300$ & $\$ 1200$ \\
\hline Summit Layer 3 switch & 1 & $\$ 100$ & $\$ 100$ \\
\hline Total for entire lab & & & $\mathbf{\$ 1 3 0 0}$ \\
\hline
\end{tabular}

Table 2 - Equipment Costs for Entire Lab

\section{VOIP AS A CONNECTIVITY TEST}

As stated before, Ping and Traceroute are two of the most valuable tools in a network administrator's toolbox. Networking curriculums rely heavily on these tools as a means of demonstrating to students that they have successfully achieved connectivity across a given local network or WAN. However, they only provide a partial picture of network connectivity, testing valid connectivity at the
Physical, Data-link, and Network layers of the OSI model (layers $1-3$ ). As the vast majority of network traffic requires successful connectivity at the Transport and Application layers (4 and 7, respectively) to properly work, a breakdown at either is not apparent when testing with Ping. Relying on these tools solely to verify connectivity omits the upper layers in testing. Applying VoIP technology for testing connectivity, such as verifying network A and network $\mathrm{B}$ can communicate by placing a telephone call between them, demonstrates to the student that the all seven OSI layers are working properly. In addition, it provides the student with a more tangible and exciting experience than simply watching the number of exclamation points from using Ping at a command prompt. As stated, Ping is a very valuable tool and is critical for use in troubleshooting network connectivity. The authors believe supplementing it with VoIP for connectivity demonstration and verification will produce a better rounded networking student.

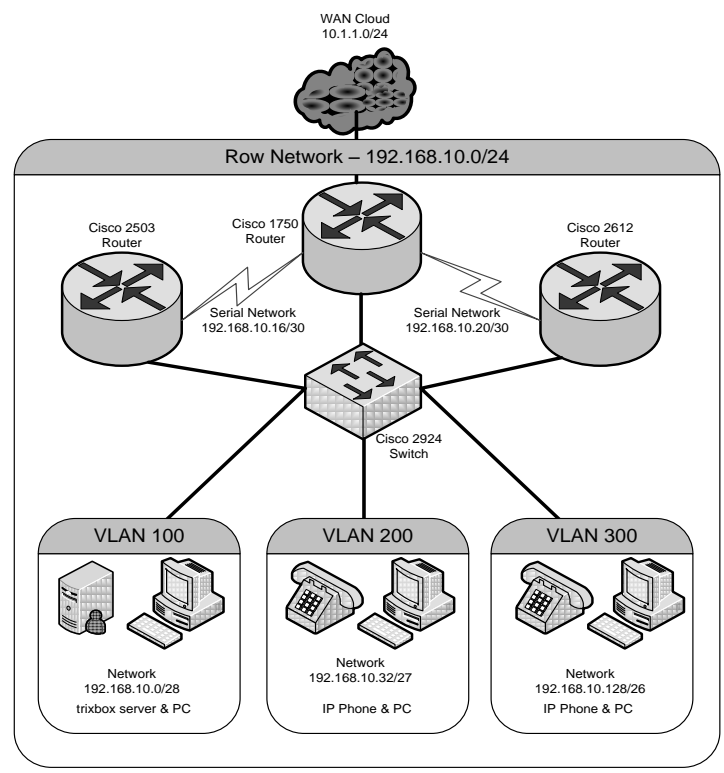

Figure 3 - Row Subnetwork Configuration

\section{STUDENT FEEDBACK}

Students who have used the lab during their coursework have found the addition of VoIP to be a new and exciting technology. The results of an informal anonymous survey have reinforced this. Remarks from students exposed to the lab environment include:

- "VoIP is a technology every IST student should be familiar with." 
- "Having VoIP knowledge, even if it is rudimentary, will help us be more marketable in the future."

- "Using the VoIP system in class is very beneficial. It provides us with a real world business setting in the class room opposed to simply theory of setting up routers."

\section{THE LOW-COST LAB AND THE SIGITE IT NETWORKING GUIDELINES}

As mentioned in the literature review, the SIGITE IT Guidelines document [2], identifies six knowledge areas of networking recommended for teaching in an IT curriculum. Using the lab environment discussed in this paper an instructor can demonstrate components from each of the six networking units. Table 3 provides examples of methods or tasks for teaching selected objectives of the SIGITE networking knowledge area.

\begin{tabular}{|l|l|}
\hline Knowledge Area & Exercise \\
\hline Foundations & Topology discussion \\
& Ethernet deployment \\
Protocol examination: & TCP, IP, SIP, IAX, ICMP \\
& OSI Layers with VoIP \\
\hline Routing and Switching & $\begin{array}{l}\text { Static routing } \\
\text { Routing with RIP } \\
\text { Layer 2 VLANs } \\
\text { Subnetting }\end{array}$ \\
\hline Physical Layer & $\begin{array}{l}\text { Cabling and connectors } \\
\text { Network bandwidth } \\
\text { Wireless }\end{array}$ \\
\hline Security & $\begin{array}{l}\text { ACLs to allow VoIP } \\
\text { RIP authentication } \\
\text { SIP security }\end{array}$ \\
\hline Network Management & $\begin{array}{l}\text { Network device config } \\
\text { Managing trixbox server } \\
\text { Examining network stats }\end{array}$ \\
\hline Application Areas & Modify config by web \\
& Examine call record data \\
\hline
\end{tabular}

Table 3 - SIGITE Networking Exercises

\section{FUTURE WORK}

Future work with this project includes the creation of a more formal and quantitative survey examining student networking knowledge before utilizing the lab and determine whether using the low-cost lab environment provides improved learning when compared to using traditional tools such as Ping for connectivity testing and demonstration. A control group of students taking a networking course will be compared to those using the lab. A pre and post test will be given to evaluate whether students retain networking knowledge better when using the designed lab in conjunction with VoIP.

\section{CONCLUSION}

As more and more students become interested in the field of networking and telecommunications, it is important that universities continue to find interesting and useful ways to instruct students. Providing them with functional equipment does not have to break the bank. We have shown that it is entirely possible to create a realistic and functional laboratory using freely available and open source software in conjunction with inexpensively sourced hardware and still being able to effectively teach networking per the SIGITE IT 2008 Guidelines.

\section{REFERENCES}

1. Abbott-McCune, S., Newtson, A. J., Girard, J., \& Goda, B. S. (2008). Developing a reconfigurable network lab. SIGITE '08: Proceedings of the 9th ACM SIGITE conference on Information technology education, 255-258.

2. ACM. (2008). Information technology 2008: Curriculum guidelines for undergraduate degree programs in information technology. http://www.acm.org/education/curricula/IT2008 $\%$ 20Curriculum.pdf

3. Cigas, J. (2002). A computer networking laboratory for administration and networking. Frontiers in Education, Annual, 1, T3D 12-11.

4. Hansen, R. A., Barlow, V. M., Gonzales, R., Rodriguez, D., \& Maza, L. (2009). A novel ip telephony course using open-source software. SIGITE '09: Proceedings of the 10th ACM conference on SIG-information technology education, 43-49.

5. Mengel, S., \& Bowling, C. (1995). Supporting networking courses with a hands-on laboratory. Frontiers in Education, Annual, 2, 20-23.

6. Yuan, D., \& Zhong, J. (2008). Designing a comprehensive open network laboratory courseware. J. Comput. Small Coll., 24 (1), 174181.

7. Yuan, D., \& Zhong, J. (2009). An instructional design of open source networking laboratory and curriculum. SIGITE '09: Proceedings of the 10th ACM conference on SIG-information technology education , 37-42. 